\section{Intoxicação por chumbo e saúde infantil: ações intersetoriais para o enfrentamento da questão}

\author{
Lead poisoning and child health: \\ integrated efforts to combat this problem
}

\author{
1 Faculdade de Medicina \\ de Botucatu, Universidade \\ Estadual Paulista Júlio \\ de Mesquita Filho, \\ Botucatu, Brasil. \\ 2 Departamento de Saúde \\ Coletiva, Secretaria \\ Municipal de Saúde \\ de Bauru, Bauru, Brasil. \\ 3 Serviço de \\ Geoprocessamento, \\ Departamento de Água \\ e Esgoto de Bauru \\ Bauru, Brasil. \\ 4 Faculdade de Odontologia \\ de Bauru, Universidade \\ de São Paulo, Bauru, Brasil. \\ 5 Outros membros listados \\ no fim do artigo. \\ Correspondência \\ N. E. Tomita \\ Departamento de \\ Odontopediatria, Ortodontia \\ e Saúde Coletiva, Faculdade \\ de Odontologia de Bauru, \\ Universidade de São Paulo. \\ Al. Dr. Octávio Pinheiro \\ Brisolla 9-75, Bauru, SP \\ 17012-101, Brasil. \\ netomita@usp.br
}

\begin{abstract}
An epidemiological survey was carried out by technicians of the State Health Secretary and the Municipal Health Secretary of Bauru, São Paulo, Brazil, due to excessive atmospheric lead emissions caused by a battery manufacturer. This survey included 853 children from 0 to 12 years old, in a 1,000-meter area from the polluting source, in Bauru (2002). The blood lead levels of children in the exposed group were higher than those in the control group ( $p<0.05) .314$ children were found to have dosages equal or superior to $10 \mu \mathrm{g} / \mathrm{dl}$, the limit stipulated by Centers for Disease Control and Prevention. Public services, universities, and volunteers developed some activities aiming at child diagnosis and treatment. The Municipal Health Secretary coordinated remediation initiatives such as: scraping the superficial surface of streets, internal aspiration of houses with professional equipment, and washing and sealing tanks. Through this work, the Lead Poisoning Study and Research Group (GEPICCB) shares an integrated, interdisciplinary, and interinstitutional action proposal.
\end{abstract}

Intersectorial Actions; Child Welfare; Lead Poisoning
Niura Aparecida de Moura Ribeiro Padula ${ }^{1}$ Maria Helena de Abreu ${ }^{2}$ Luís César Yoshinori Miyazaki 3 Nilce Emy Tomita 4 Grupo de Estudo e Pesquisa da Intoxicação por Chumbo em Crianças de Bauru 5

\section{Introdução}

Rojas et al. 1 referem que, embora a exposição ao chumbo esteja em declínio nos países em desenvolvimento, a contaminação crônica por exposição a baixos níveis continua sendo um problema importante para a saúde infantil.

As crianças apresentam um elevado risco à exposição ao chumbo, sendo especialmente suscetíveis aos seus efeitos tóxicos 2,3,4. O Fundo da Nações Unidas para a Infância (UNICEF) 5 reafirma a vulnerabilidade infantil ao chumbo, devido aos maiores níveis de absorção desse metal pesado, quando comparados aos adultos. Os sistemas nervoso e digestivo da criança são especialmente suscetíveis a alterações e, adicionalmente, a criança tende a explorar o mundo com a boca, entrando em contato com o chumbo presente no solo, na poeira doméstica e no ar.

Os mecanismos pelos quais a exposição ao chumbo ocasiona esses agravos à saúde foram alvo de um estudo de Moreira \& Moreira 6, que consideram que seus efeitos nocivos podem afetar praticamente todos os órgãos e sistemas do organismo humano, com base em vários modelos cinéticos que tentam explicar a distribuição do chumbo no organismo.

Tendo em vista que a importância global suscitada pela contaminação por chumbo implica compreender o problema sob os aspectos da desigualdade 1 , vulnerabilidade 7 , processo de adoecimento e capacidade de resposta do 
sistema social, este estudo visa a contribuir para a discussão da complexa questão que envolve a saúde e o ambiente.

\section{Breve histórico}

A Companhia de Tecnologia de Saneamento Ambiental (CETESB) notificou a Secretaria de Estado da Saúde de São Paulo (SES-SP) e a Secretaria Municipal de Saúde de Bauru (SMSBauru), em janeiro de 2002, quanto à interdição de uma indústria de acumuladores, em função da emissão de partículas de chumbo para o meio ambiente acima do permitido ${ }^{8}$. A partir da localização desta fábrica, instalada desde 1958 em área periférica do município, dejetos contendo sal de óxido de chumbo e sulfato de chumbo presentes na poeira e a deposição de chumbo metálico no solo foram detectados 8,9 .

O Grupo de Vigilância Epidemiológica (GVE/ SES-SP) e o Grupo de Vigilância Sanitária (GVS/ SES-SP) sediados na Direção Regional de Saúde de Bauru (DIR-X/SES-SP), com suporte técnico da Divisão de Doenças Ocasionadas pelo Meio Ambiente (DOMA/SES-SP), Centro de Vigilância Epidemiológica (CVE/SES-SP) e Centro de Vigilância Sanitária (CVS/SES-SP), em conjunto com a SMS-Bauru, elaboraram um plano para verificar as fontes de exposição ao chumbo, a exposição humana a esse metal pesado e as necessidades de intervenção em relação à saúde humana e ao meio ambiente.

Para avaliar a exposição populacional ao chumbo, optou-se por estudar as crianças, consideradas um grupo de maior risco 2,5.

Inicialmente, foram colhidas amostras sangüíneas para dosagem de chumbo em dois grupos de crianças de 2 a 7 anos de idade. $O$ grupo exposto foi formado por 29 crianças, moradoras na região há mais de quatro anos ou que nasceram no local, e o grupo-controle, por 31 crianças residentes em distância superior a $11 \mathrm{~km}$ da empresa e com características sociais semelhantes ao grupo exposto.

A análise laboratorial foi realizada no Instituto Adolfo Lutz de São Paulo (IAL/SES-SP). Os níveis de plumbemia no grupo exposto tiveram valor médio de $7,72 \mu \mathrm{g} / \mathrm{dl}$ e eram mais elevados que os do grupo-controle, cujos valores estavam abaixo do limite de detecção do método, de $5 \mu \mathrm{g} / \mathrm{dl}(\mathrm{p}<0,05)$. Estabeleceu-se como limite de intervenção o nível de plumbemia igual ou superior a $10 \mu \mathrm{g} / \mathrm{dl}$, segundo critérios do Centers for Disease Control and Prevention (CDC) 2 .

Esses resultados indicaram a necessidade de aprofundar a investigação, realizando um estudo transversal visando a avaliar as condi- ções de todas as crianças de 0 a 12 anos residentes em área próxima à empresa.

Mediante a necessidade da participação dos serviços públicos e seus gestores, com apoio de instituições de ensino e pesquisa, foram envolvidos profissionais da SES/SP, SMS-Bauru, CETESB, Ministério da Saúde (MS), Fundação Jorge Duprat Figueiredo de Segurança e Medicina do Trabalho (FUNDACENTRO), Centro de Intoxicações da Faculdade de Ciências Médicas da Universidade Estadual de Campinas (FCM-UNICAMP), Faculdade de Medicina de Botucatu da Universidade Estadual Paulista Júlio Mesquita Filho (FMB-UNESP), Faculdade de Ciências (FC-UNESP/Bauru), Faculdade de Odontologia de Bauru da Universidade de São Paulo (FOB-USP) e Hospital de Reabilitação de Anomalias Craniofaciais (HRAC-USP). Seus representantes compõem o Grupo de Estudo e Pesquisa da Intoxicação por Chumbo em Crianças de Bauru (GEPICCB), que desencadeou medidas de investigação clínico-epidemiológica e tratamento das crianças.

\section{Método}

\section{Pesquisa de campo}

Foi definido um raio de $1.000 \mathrm{~m}$ ao redor da empresa como área inicial de estudo, buscando identificar todas as crianças de 0 a 12 anos (Figura 1). Seus pais/responsáveis foram entrevistados em visitas domiciliares, por técnicos da SMS-Bauru, investigando fatores de exposição ao chumbo no local e outras possíveis fontes de exposição não relacionadas à área, sendo a coleta de amostras de sangue para exame de plumbemia realizada por profissionais do IAL/SES-SP.

As visitas foram realizadas com viatura cedida pela empresa. Todos os participantes foram informados sobre a finalidade do levantamento e seus responsáveis apresentaram consentimento por escrito. Inicialmente, observaram-se recusas, que foram decrescendo e sendo revertidas ao longo da realização dos trabalhos.

Foram entrevistadas e tiveram material coletado para exame laboratorial 883 pessoas residentes nessa área, totalizando 857 crianças, duas gestantes, 23 nutrizes e um adulto, sendo esses procedimentos finalizados em janeiro de 2003.

\section{Conduta clínica e vigilância epidemiológica}

Os exames laboratoriais detectaram 314 crianças cujos índices de plumbemia indicavam exposição elevada a chumbo. Esses resultados 

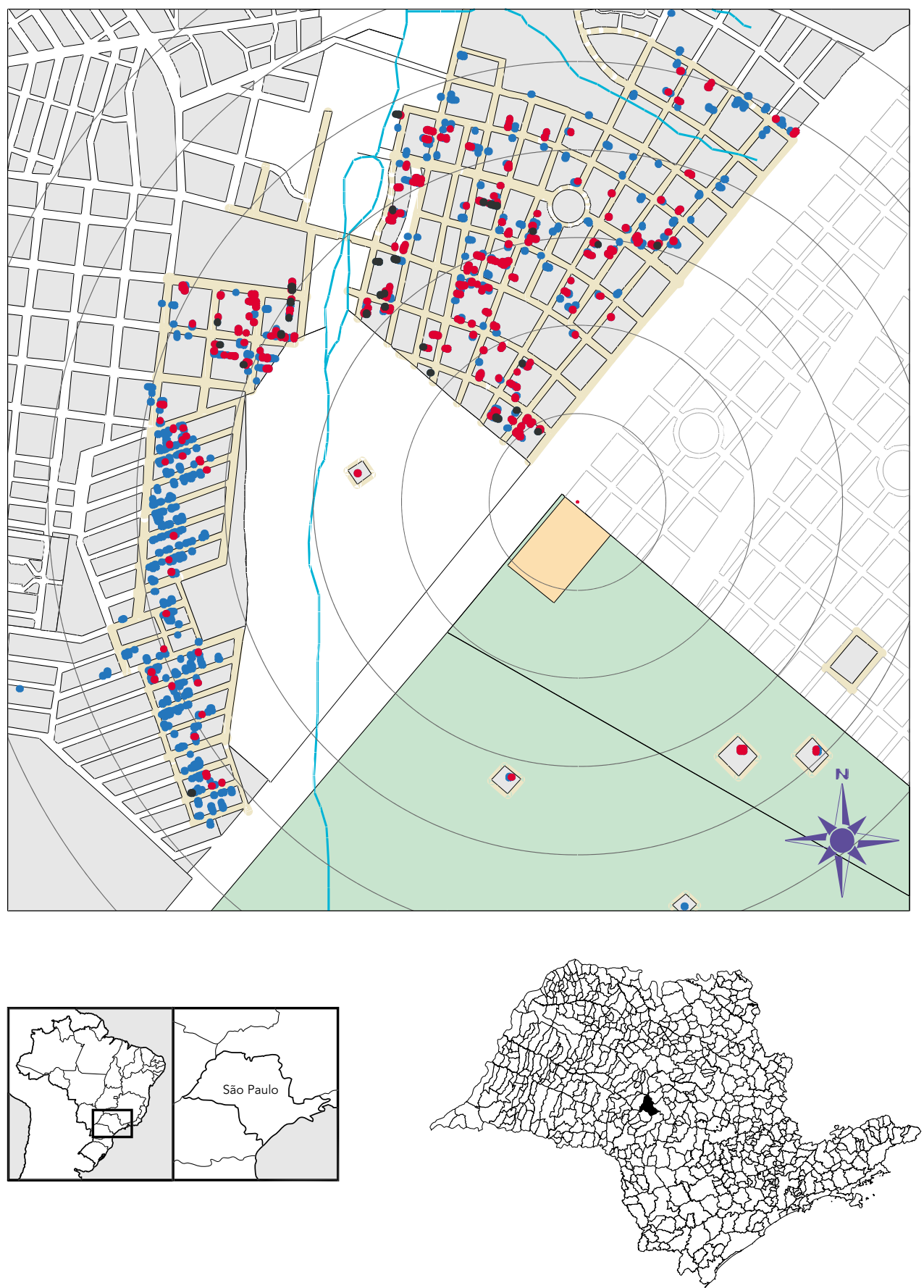

Resultado da plumbemia das crianças

área de proteção ambiental

rios e córregos

quadras habitadas

quadras desabitadas

fonte de contaminação

raios de $200 \mathrm{~m}$

crianças: < 10 $\mu \mathrm{g} / \mathrm{dl}$

crianças: $\geq 10$ e $<25 \mu \mathrm{g} / \mathrm{d}$ l

crianças: $\geq 25 \mu \mathrm{g} / \mathrm{dl}$ 
nortearam ações de avaliação clínica, laboratorial e radiográfica das crianças.

Os procedimentos clínicos, em andamento, são realizados sob a coordenação da equipe de Neuropediatria da FMB-UNESP, por equipe multiprofissional composta de médicos (otorrinolaringologistas, pediatras, hematologistas), dentistas, fonoaudiólogos, psicólogos, fisioterapeutas, assistentes sociais e auxiliares. A avaliação inclui os seguintes exames complementares: radiografias panorâmicas de face, de ossos longos e carpal para determinação da idade óssea, eletrocardiograma (ECG), eletroencefalograma (EEG), audiometria, avaliação laboratorial das funções hepática e renal, hemograma e ferro sérico para todas as crianças.

Para as crianças cujo resultado inicial de plumbemia foi igual ou superior a $25 \mu \mathrm{g} / \mathrm{dl}$, foi realizado o exame complementar de eletroneuromiografia, mediante a suspeita de alterações neurológicas importantes. $\mathrm{O}$ atendimento a estas situações, consideradas mais graves, foi priorizado, tendo estas crianças recebido o tratamento à base de quelação por EDTA, o que requereu internação hospitalar para observação e acompanhamento em 23 casos.

Todas as crianças com taxas de plumbemia acima do aceito pelo CDC 2 receberam avaliação neuropediátrica, com exceção de duas que foram a óbito por outras causas, cinco se mudaram de Bauru e duas cujos responsáveis optaram por assistência privada. Os achados clínicos resultantes destes exames são apresentados em outra publicação.

A empresa colabora com o processo, providenciando transporte e alimentação para as crianças e seus acompanhantes e fornecendo alguns materiais de consumo para exames.

\section{Conduta em vigilância sanitária}

O Departamento de Saúde Coletiva (SMS-Bauru), sob orientação técnica do GVS/SES-SP, coletou amostras de hortifrutigranjeiros, água e leite da região, com análise feita pelo IAL/ SES-SP.

Em fevereiro de 2002, ovos, hortelã e leite in natura apresentaram concentrações de chumbo acima dos valores aceitos pela Organização Mundial da Saúse (OMS), enquanto as amostras de água estavam satisfatórias.

Em abril de 2002, coletas de almeirão, ovos, leite in natura e mandioca apresentaram níveis de chumbo acima do tolerado, em algumas localidades, enquanto amostras de cebolinha, hortelã, alface, salsa, ovos e mandioca de outras chácaras estavam satisfatórias. É importante ressaltar o cuidado com esses exames, uma vez que essas amostras satisfatórias foram encontradas em chácara que abastece o mercado local.

Seguindo recomendação do CVS/SES-SP, que levou em consideração análises de todos os resultados de alimentos, água e solo, recomendouse restrição ao consumo de alimentos da área. Na Figura 2, o mapa temático mostra a contaminação do solo (até $2 \mathrm{~cm}$ de profundidade) com base nos dados fornecidos pela CETESB.

O monitoramento dos alimentos prosseguiu, com os resultados de setembro de 2002 mostrando que os ovos e o leite in natura caprino, embora satisfatórios, estavam próximos ao limite, e o leite in natura bovino apresentava-se insatisfatório.

Utilizando ferramentas de geoprocessamento, os dados epidemiológicos, ambientais e geológicos puderam ser localizados espacialmente. A técnica de georreferenciamento possibilitou a visualização de casos no território definido para o estudo e a partir da inserção destas informações foram gerados os mapas temáticos.

\section{Resultados}

Foram detectadas 314 crianças com níveis de plumbemia igual ou superior a $10 \mu \mathrm{g} / \mathrm{dl}$. A Figura 1 utiliza ferramentas de geoprocessamento para localizar espacialmente estas crianças, categorizando as crianças intoxicadas em dois grupos: um grupo com taxas até $24 \mu \mathrm{g} / \mathrm{dl}$ e o segundo com taxas acima de $25 \mu \mathrm{g} / \mathrm{dl}$. Crianças com taxas inferiores a $10 \mu \mathrm{g} / \mathrm{dl}$ são visualizadas em maior número em áreas que apresentam asfaltamento das ruas.

Na Figura 3, observa-se o uso de um mapa temático no planejamento de ações emergenciais. A partir da localização de casos, segundo a gravidade expressa pelos níveis de plumbemia, foi estabelecido um roteiro de trabalho contemplando prioritariamente as ruas e domicílios que continham crianças intoxicadas por chumbo. O trabalho de raspagem do solo superficial obedeceu à seqüência: (a) ruas onde residiam crianças com índices acima de $25 \mu \mathrm{g} / \mathrm{dl}$ de chumbo no sangue, (b) áreas com crianças com índices entre 10 e 25 $\mathrm{gg} / \mathrm{dl}$ e (c) áreas com crianças cujos níveis estavam abaixo de $10 \mu \mathrm{g} / \mathrm{dl}$. O fator distância da fonte emissora também foi considerado nas ações de campo.

O trabalho de campo foi coordenado pela SMS-Bauru, sendo executado por trabalhadores da empresa e monitorado pela CETESB, que autorizou a deposição de terra resultante das raspagens de solo superficial em um galpão cimentado e coberto nas dependências da empresa. 


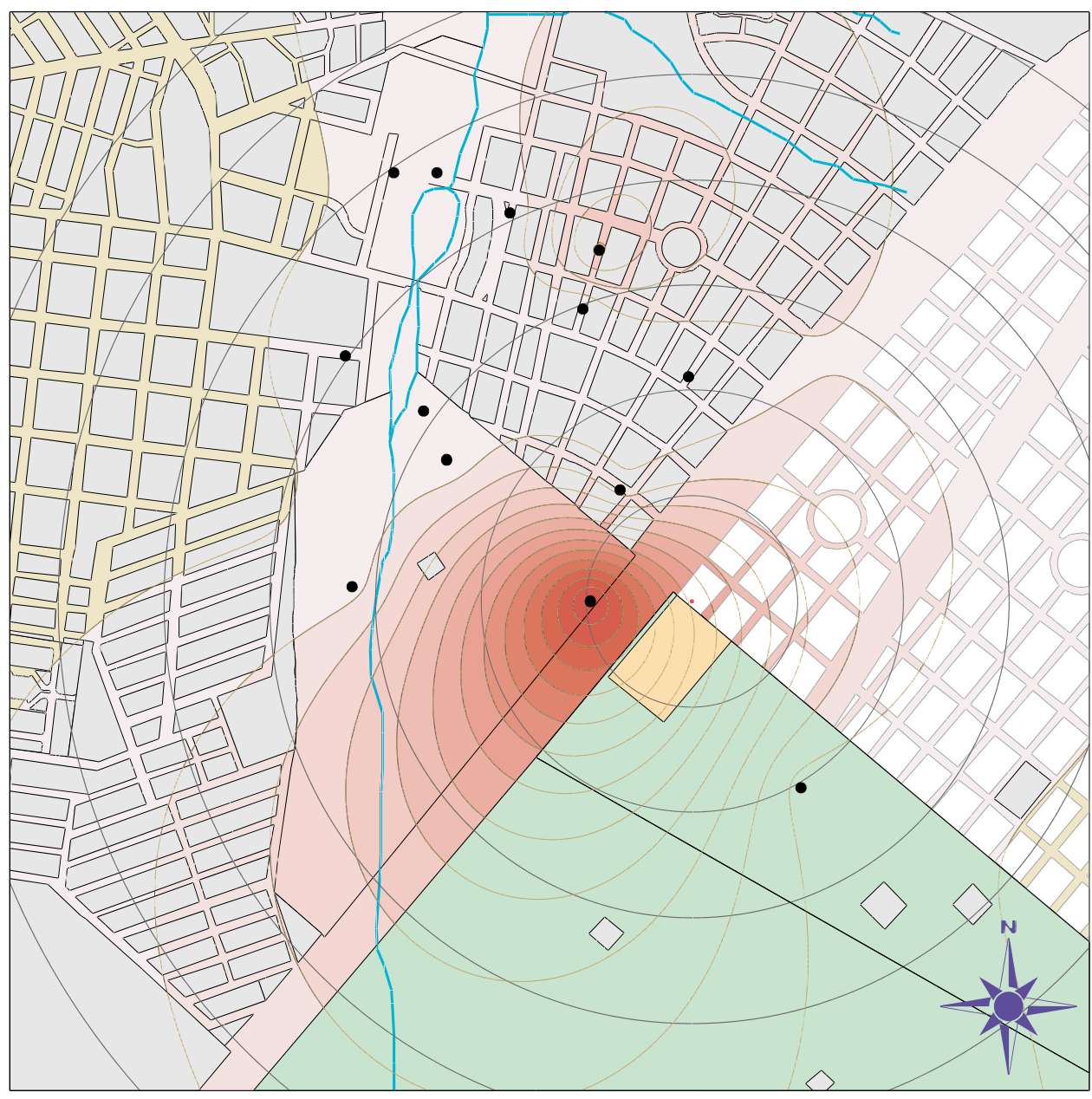

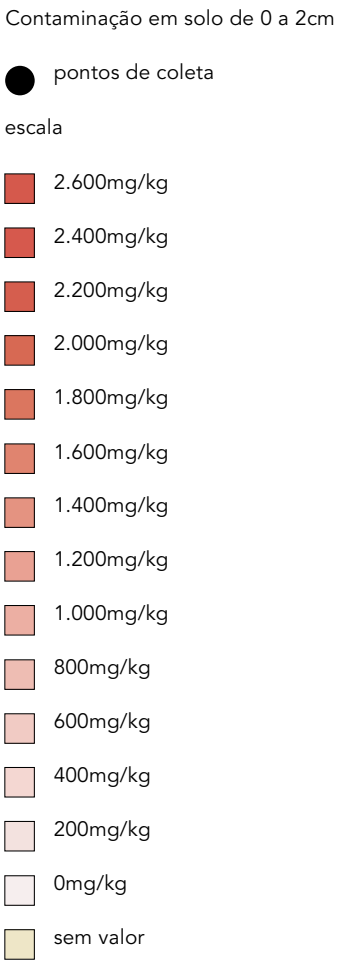

Algumas ações de saneamento foram conduzidas pela SMS-Bauru, Secretaria Municipal de Obras (SMO), Secretaria Municipal de Meio Ambiente (SEMMA), Secretaria Municipal das Administrações Regionais (SEAR) e Departamento de Água e Esgoto (DAE).

Em reunião com os moradores da área, a SMS-Bauru promoveu orientações para o saneamento das habitações, uma vez que as medidas de saneamento do meio ambiente nas áreas contaminadas, em especial, locais peri e intradomiciliares, ficaram a cargo do poder público municipal.

Os trabalhos de campo foram realizados de novembro de 2002 a janeiro de 2003, com 28 recusas para aspiração do interior dos domicílios e raspagem de quintais (Tabela 1).

\section{Discussão}

Em nível nacional, outros eventos adversos envolvendo a contaminação ambiental por chumbo têm sido relatados, como os ocorridos no Vale do Ribeira, Luanda (Paraná), Jacareí (São Paulo) e Santo Amaro da Purificação (Bahia). Existe alguma dificuldade em realizar uma estimativa de exposição ao chumbo no Brasil, considerando a existência de atividades informais e de passivos ambientais. A população com exposição não ocupacional tem sido identificada em raios de $500 \mathrm{~m}$ da fonte, quando se trata de pequenas fundições, e $1.000 \mathrm{~m}$ da fonte, para as fundições de médio e grande porte. Os grupos de maior risco são constituídos por crianças residentes no entorno de emissões de 


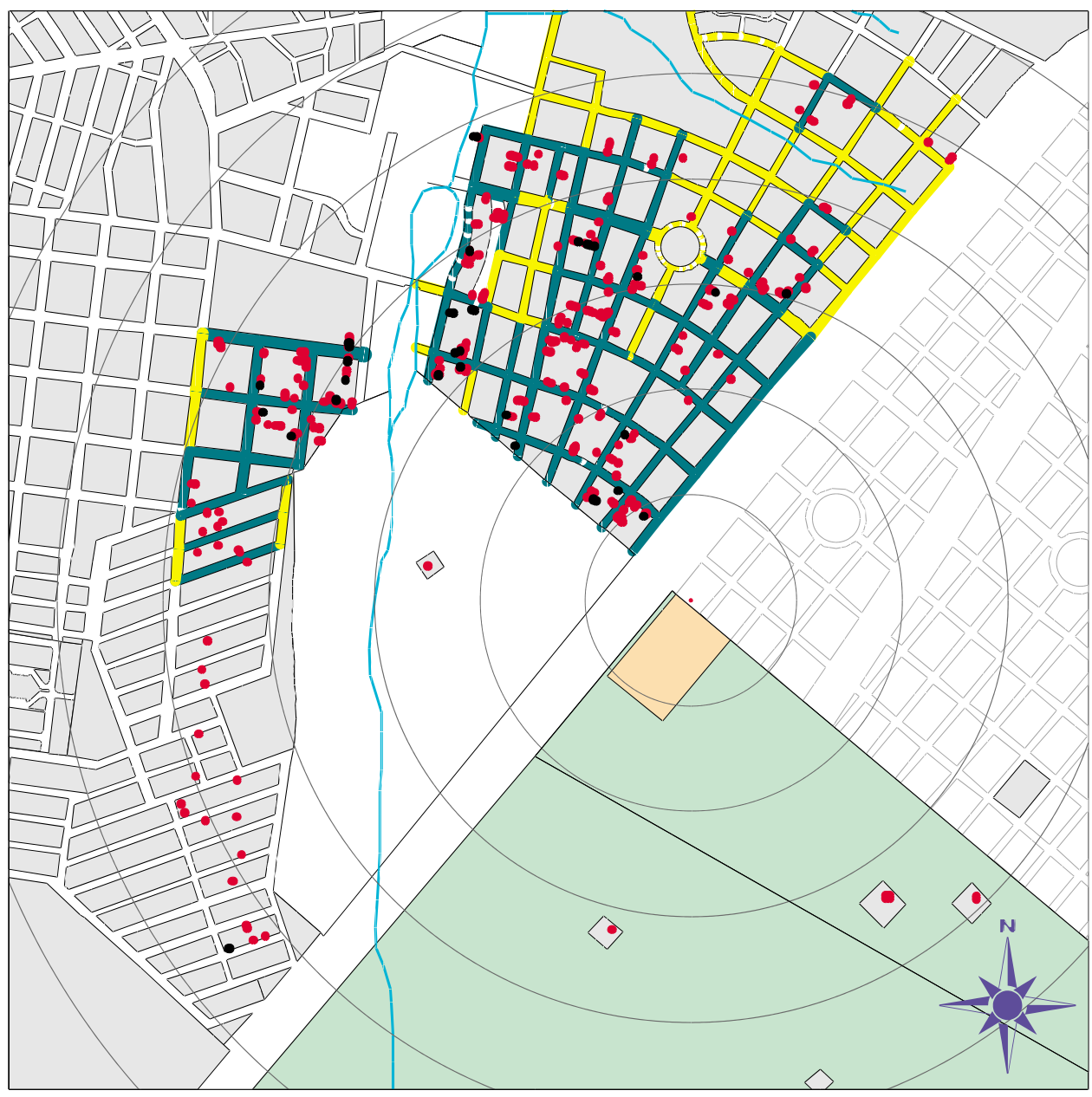

Trabalhos realizados

área de proteção ambiental

rios e córregos

quadras habitadas

quadras desabitadas

fonte de contaminação

raios de $200 \mathrm{~m}$

locais sem crianças contaminadas

locais raspados e aspirados

crianças: $\geq 10$ e $<25 \mu \mathrm{g} / \mathrm{d}$

crianças: $\geq 25 \mu \mathrm{g} / \mathrm{dl}$ chumbo e gestantes, considerando a sua suscetibilidade intrínseca (Trivelato GC, Asmus CIRF, Freitas C, Alonzo H, Simonetti MH, Paoliello MMB, et al. Termo de referência do Grupo de Trabalho Chumbo. Oficina para estruturação das ações de vigilância ambiental em saúde relacionada às substâncias químicas: amianto, benzeno, mercúrio, agrotóxicos e chumbo. Proposta de modelo de atuação, promovida pela Coordenação Geral de Vigilância Ambiental/Ministério da Saúde; 2004).

Globalmente, registram-se ocorrências no México 10,11, Suécia 12, Estados Unidos 13, Uruguai 14, Portugal 15, Índia 16 e Venezuela 1, com particular destaque sobre as repercussões dos teores elevados de chumbo na saúde infantil.
A mitigação de problemas ambientais tem sido o foco de alguns relatos na literatura internacional. Destacam-se os casos polares de Seveso, Itália e Bhopal, Índia. De Marchi 17 (p. 41), ao comentar o acidente de Seveso, em que houve liberação de uma nuvem tóxica contendo dioxina proveniente de uma indústria química, refere que "ninguém na cidade sabia que estava em situação de risco, não havendo consciência do problema, tanto por parte da população local, como também por parte das autoridades públicas, incluindo a sanitária". "Se em muitos aspectos os acidentes podem ter conseqüências semelhantes (...), as diferenças maiores se encontram na resposta da sociedade a esse tipo de acidente. Essas diferenças revelam a vulnerabi- 
Atividades emergenciais efetuadas no Bairro Jardim Tangarás. Bauru, São Paulo, Brasil, 2002/2003.

\begin{tabular}{|c|c|c|c|}
\hline Atividades & Alvo & Desfecho & Realizado/supervisionado por \\
\hline $\begin{array}{l}\text { Cimentação de piso no } \\
\text { interior das residências }\end{array}$ & Moradias com piso de terra & 3 residências & Secretaria Municipal de Obras de Bauru \\
\hline $\begin{array}{l}\text { Raspagem de } 5 \mathrm{~cm} \text { de solo } \\
\text { superficial em ruas de terra }\end{array}$ & 80 ruas & & $\begin{array}{l}\text { Empresa/Secretaria Municipal de Obras de Bauru/ } \\
\text { Secretaria Municipal de Saúde de Bauru/ } \\
\text { Secretaria Municipal de Meio Ambiente de Bauru }\end{array}$ \\
\hline $\begin{array}{l}\text { Raspagem de } 5 \mathrm{~cm} \text { de solo } \\
\text { superficial dos quintais }\end{array}$ & 270 residências & & $\begin{array}{l}\text { Empresa/Secretaria Municipal de Saúde de Bauru/ } \\
\text { Secretaria Municipal de Meio Ambiente de Bauru }\end{array}$ \\
\hline $\begin{array}{l}\text { Aspirações de interiores } \\
\text { de residências }\end{array}$ & $\begin{array}{l}\text { Residências sem forros/ } \\
\text { muito próximas à empresa }\end{array}$ & 164 residências & $\begin{array}{l}\text { Empresa/Secretaria Municipal de Meio } \\
\text { Ambiente de Bauru }\end{array}$ \\
\hline $\begin{array}{l}\text { Limpeza e vedação } \\
\text { de caixas d'água }\end{array}$ & $\begin{array}{l}\text { Moradias sem vedação } \\
\text { de caixas d'água }\end{array}$ & $\begin{array}{l}177 \text { visitas } \\
\text { e } 82 \text { limpezas }\end{array}$ & Departamento de Água e Esgoto/Empresa \\
\hline
\end{tabular}

lidade das populações em termos da capacidade de resposta no sistema social, que ou protege as pessoas (...), como foi o caso de Seveso, ou abandona as mesmas a sua própria sorte, como foi o caso de Bhopal" 17 (p. 45).

Neste aspecto, a relação entre saúde e ambiente vai sendo moldada pela intervenção de alguns setores do sistema social, visando a reverter o processo de adoecimento das crianças sob risco. Em nível local, a experiência envolvendo o conjunto de ações intersetoriais relatadas teve espaço em um contexto social e ambiental amplamente desfavorável.

A escassez de fontes de referência que contivessem a indicação de protocolos clínicos, ambientais, administrativos e epidemiológicos foi detectada nos momentos iniciais do enfrentamento desta questão de saúde pública.

O tom alarmista adotado inicialmente por alguns veículos de comunicação colocou a população diante de um problema desconhecido. Na construção da relação entre os setores, a imprensa mostrou um papel de mediação, divulgando fatos, expondo inquietações e dando voz aos diversos segmentos. Por um lado, pode-se perceber a atuação da imprensa mostrando os conflitos, e por outro, constituindo um veículo para uma espécie midiática de controle social 18.

Focada pelo olhar da biopolítica, modalidade de exercício do poder que garante a sobreposição entre vida e política, e que "possibilita que com um mesmo gesto sejam definidas as populações que pertencem ao espaço da vida nua e aquelas que fazem parte da vida ativa, isto é, da condição humana que deve ser cuidada, estimulada, multiplicada" 19 (p. 454), a opção realizada coletivamente foi pela atenção às demandas desse grupo populacional exposto a um agravo socioambiental.
O espaço de negociação para esta reflexão envolveu articulações que demandaram ampla participação do nível central, representado por gestores estaduais e do nível local, representado por gestores e trabalhadores do município, com importantes articulações com o nível federal. Os segmentos constituídos por técnicos, formadores e pesquisadores foram sendo agregados, na medida em que a ausência de protocolos demandava decisões de caráter técnicocientífico, para o qual a rede de serviços públicos não tinha respostas imediatas.

Iniciativas individuais ou de interesse essencialmente acadêmico foram desestimuladas desde o primeiro momento, a partir do foco na necessidade de decisões políticas visando à condução de ações que priorizassem o diagnóstico da situação e o atendimento das crianças expostas/intoxicadas.

Algumas limitações para o enfrentamento da vulnerabilidade da população exposta 7 têm sido observadas. A escassez de informações sobre o que fazer no município induziu um processo de tentativas baseadas em critérios clínicos no que se refere à assistência às crianças, $\mathrm{e}$ no senso comum e árduo trabalho de campo, no que tange às ações ambientais, destacando o importante papel do poder público e a colaboração da empresa.

Ao realizar previsões e tomar decisões num contexto de incertezas, de riscos tecnológicos, ambientais e estruturais, "Hottois propõe uma ética de solidariedade (...), baseada (...) no diálogo aberto, que implica o confronto pluralista e interdisciplinar; na ética reguladora; no pragmatismo; na não-exclusão do sentimento (...) do conjunto de elementos que cooperam na tomada de decisão ética; na ética da ambivalência, no sentido de ser esta uma escolha, e não uma con- 
clusão lógica, ou um resultado mecânico; na ética evolutiva e da reversibilidade dos princípios; na ética da co-responsabilidade" 20 (p. 363).

Moreira \& Moreira 6 (p. 178) sugerem que " $a$ percepção da série de eventos entre a exposição ao chumbo e as alterações biológicas que levam a efeitos adversos é fundamental para o desenvolvimento de métodos relevantes de monitoramento biológico", o que pode vir a constituir uma contribuição do follow-up proposto pelo GEPICCB.

\section{Considerações finais}

Quanto à descontaminação definitiva da área, a CETESB está encaminhando à empresa um plano de ações resolutivas, para que a região possa ser habitada sem intercorrências futuras, ressaltando que a empresa continua interditada por tempo indeterminado. O DAE realiza o mapeamento da região, para monitoramento de eventuais contaminações no lençol freático da área atingida.

Algumas dificuldades decorrem das condições de existência da população focada neste relato, amplificando sua vulnerabilidade. Aspectos importantes relativos ao acesso a bens essenciais, como alimentação adequada, moradia condizente, serviços de atenção básica à saúde, alfabetização de pais/responsáveis, educação infantil, saneamento básico, bem como alguma modalidade de organização popular, extrapolam as possibilidades de intervenção imediata sobre essa população de risco.

Nos espaços de negociação criados, novos diálogos entre os diversos setores são necessários, para além das ações emergenciais de suporte e assistência à saúde. O compartilhamento de experiências constitui uma parte da construção deste processo de trabalho, em que tecnologias consideradas "leves" têm tanto peso quanto aquelas consideradas “duras” (Merhy, 1997, apud Capozollo et al. 21).
Resumo

Inquérito epidemiológico realizado pela Secretaria de Estado da Saúde de São Paulo e Secretaria Municipal de Saúde de Bauru visou à realização de exames de plumbemia em 853 crianças de 0 a 12 anos, em Bauru, São Paulo, Brasil (2002), a partir de indícios de chumbo oriundo de resíduos industriais nas proximidades de uma fábrica de baterias. Os níveis sangüineos de chumbo no grupo controle foram inferiores aos apresentados pelo grupo exposto $(p<0,05)$. Mediante a existência de 314 crianças com taxas de plumbemia superiores àquelas aceitáveis pelo Centers for Disease Control and Prevention (10 $\mu \mathrm{gPb} / \mathrm{dl}$ sangue), foi desencadeado um conjunto de ações com participação dos serviços públicos, universidades e voluntariado, para promover o diagnóstico e a assistência à saúde da população atingida. Ações emergenciais, visando a reduzir riscos de recontaminação, incluíram a raspagem de camada superficial das vias públicas, resultando em $1.392 m^{3}$ de terra contendo material tóxico, que permanece depositada nas dependências da fábrica. Foi promovida a aspiração de poeira do interior das residencias e a lavagem e vedamento das caixas d'água. O Grupo de Estudo e Pesquisa da Intoxicação por Chumbo em Crianças de Bauru, por meio deste trabalho, faz o compartilhamento de uma experiência intersetorial, multidisciplinar e interinstitucional.

Ação Intersetorial; Bem-estar da Criança; Intoxicação por Chumbo

\section{Colaboradores}

N. A. M. R. Padula participou da discussão dos achados. M. H. Abreu participou da coleta de dados e discussão dos achados. L. C. Y. Miyazaki contribuiu no geoprocessamento de dados e discussão dos achados. N. E. Tomita contribuiu no planejamento do estudo, discussão e redação.

\section{Outros membros do Grupo de Estudo e Pesquisa da Intoxicação por Chumbo em Crianças de Bauru}

K. F. Alvarenga, J. G. T. M. Angerami, P. A. P. Crenitte, P. Ferraz, C. U. Freitas, J. A. S. Freitas, J. R. P. Lauris, M. M. F. Lima, C. H. F. Martins, E. Miranda, T. M. Ribeiro, O. M. P. R. Rodrigues, M. H. Simonetti. 


\section{Referências}

1. Rojas M, Espinosa C, Seijas D. Asociación entre plomo en sangre y parámetros sociodemográficos en población infantil. Rev Saúde Pública 2003; 37:503-9.

2. Centers for Disease Control and Prevention. Preventing lead poisoning in young children. Atlanta: Department of Health and Human Services; 1991.

3. Lanphear BP, Roghmann KJ. Pathways of lead exposure in urban children. Environ Res 1991; 74: 67-73.

4. Tong S, Von Schimding YE, Prapamontol T. Environmental lead exposure: a public health problem of global dimensions. Bull World Health Organ 2000; 78:1068-77.

5. Fundo das Nações Unidas para a Infância. Saturnismo infantil: información para promover los intereses de la infancia y adoptar medidas. s.l.; 1997.

6. Moreira FR, Moreira JC. A cinética do chumbo no organismo humano e sua importância para a saúde. Cienc Saúde Coletiva 2004; 9:167-81.

7. Porto MFS. Entre a saúde e a vulnerabilidade: em busca de uma abordagem ecossocial em problemas de saúde e ambiente. In: Porto MFS, Freitas $\mathrm{CM}$, organizadores. Problemas ambientais e vulnerabilidade: abordagens integradoras para o campo da saúde pública. Rio de Janeiro: Escola Nacional de Saúde Pública, Fundação Oswaldo Cruz; 2002. p. 125-36.

8. Secretaria de Estado do Meio Ambiente de São Paulo. Auto de infração e imposição de penalidade de interdição temporária. Processo n. 07/00524/ 01 da CETESB: penalidade de interdição temporária por infração aos Artigos 2o, 3o, inciso V; 33 e 51 do Regulamento da Lei 997/76, aprovado pelo Decreto 8.468/76. São Paulo: Secretaria de Estado do Meio Ambiente de São Paulo; 2002.

9. Instituto Ambiental Vidágua. Nota oficial 08/04/ 2002: poluição por chumbo em Bauru. http:// www.vidagua.org.br/chumbo.htm (acessado em 16/Abr/2004).

10. Jimenez C, Romieu I, Palazuelos E, Muñoz I, Cortes M, Rivero A, et al. Factores de exposición ambiental y concentraciones de plomo en sangre en niños de la Ciudad de México. Salud Pública Mex 1993; 35:599-606.

11. Olaiz G, Fortoul TI, Rojas R, Doyer M, Palazuelos E, Tapia CR. Risk factors for high levels of lead in blood of schoolchildren in Mexico City. Arch Environ Health 1996; 51:122-6.
12. Fulton M, Raab G, Thompson G, Laxen D, Hunter $\mathrm{R}$, Hepburn W. Influence of blood level on the ability and attainment of children in Edinburg. Lancet 1987; 1:1221-6.

13. Kurtin D, Therrell B, Patterson P. Demographic risk factors associated with elevated lead levels in Texas children covered by Medicaid. Environ Health Perpect 1997; 105:66-8.

14. Shultz A, Barregard L, Sallsteen G, Wilske J, Manay N, Pereira L, et al. Blood lead in Uruguayan children and possible sources of exposure. Environ Res 1997; 74:17-23.

15. Mayan ON, Henriques AT, Calheiro JM. Childhood lead exposure in Oporto, Portugal. Int J Occup Environ Health 2001; 7:209-16.

16. Patel AB, Williams SV, Frumkin H, Kondawar VK, Glick H, Ganju AK. Blood lead in children and its determinants in Nagpur, India. Int J Occup Environ Health 2001; 7:119-26.

17. De Marchi B. Comunicação e informação de riscos: a experiência da comunidade européia com os grandes acidentes industriais. In: Porto MFS, Freitas CM, organizadores. Problemas ambientais e vulnerabilidade: abordagens integradoras para o campo da saúde pública. Rio de Janeiro: Escola Nacional de Saúde Pública, Fundação Oswaldo Cruz; 2002. p. 405-56.

18. Tomita NE, Padula NAMR; Grupo de Estudo da Intoxicação por Chumbo em Crianças de Bauru. Intoxicação por chumbo em crianças de BauruSP: análise do discurso da imprensa [CD-ROM]. Rev Bras Epidemiol 2004.

19. Caponi S. A biopolítica da população e a experimentação com seres humanos. Cienc Saúde Coletiva 2004; 9:445-55.

20. Silva ER, Schramm FR. A questão ecológica: entre a ciência e a ideologia/utopia de uma época. Cad Saúde Pública 1997; 13:355-65.

21. Capozollo A, Fortuna CM, Santos H, Un JW, Matumoto S, Resende S, et al. Curso de formação dos facilitadores de educação permanente em saúde: unidade do trabalho e relações na produção do cuidado. Brasília: Secretaria de Gestão do Trabalho e Educação na Saúde, Departamento de Gestão da Educação na Saúde, Ministério da Saúde; 2004.

Recebido em 09/Ago/2004

Versão final reapresentada em 17/Fev/2005

Aprovado em 24/Jun/2005 